\title{
Space weathering of asteroids: similarities and discrepancies between Main Belt asteroids and NEOs
}

\author{
S. Marchi ${ }^{1}$, P. Paolicchi ${ }^{2}$, M. Lazzarin ${ }^{1}$ and S. Magrin ${ }^{1}$ \\ ${ }^{1}$ Dipartimento di Astronomia, Università di Padova, Vicolo dell'Osservatorio 2, I-35122 \\ Padova, Italy \\ email: simone.marchi@unipd.it; sara.magrin.1@unipd.it; monica.lazzarin@unipd.it \\ ${ }^{2}$ Dipartimento di Fisica, Università di Pisa, largo Pontecorvo 3, I-56127 Pisa, Italy \\ email: paolicchi@df.unipi.it
}

\begin{abstract}
A sample of $35 \mathrm{C}-$ complex objects is present among near-Earth objects. In spite of the poor statistics, some striking differences compared to Main Belt asteroids can be established: for instance the percentage of near-Earth objects (NEOs) showing hydration features is very small. Moreover the spectral slope of $\mathrm{C}-$-complex NEOs seems to be anti-correlated with the exposure to the ion flux coming from the Sun, in contrast with the general behavior of C-complex Main Belt asteroids (and of most asteroids, in general). We discuss some possible implications and suggest some preliminary partial explanations.
\end{abstract}

Keywords. asteroids, surface

\section{Introduction}

In two recent papers (Marchi et al. 2006a and Lazzarin et al. 2006) it has been shown that the mean visible spectral slope of most Main Belt asteroids ( $\mathrm{S}-, \mathrm{C}$ - and $\mathrm{X}$-complexes) is positively correlated with the exposure to the weathering effects due to the Sun. These findings have relevant consequences for what concerns the chemistry, the evolutionary history and even the taxonomical classification of Main Belt asteroids (MBAs). Notice that the dominance of reddening effects within the $\mathrm{C}$-complex is effective for what concerns the bulk of MBAs, but does not hold for some subgroups, such as some families or bodies in the outer Main Belt.

Among the $\mathrm{C}-$-complex MBAs some exhibit hydration features, some others do not. This occurrence indicates the presence of some intrinsic compositional variation or the presence of some selective process - not yet well understood-in the history of C-complex asteroids; however we remark that the presence of hydration features seems to be not relevant for what concerns the properties of the space weathering: both samples redden with the exposure in a similar way.

\section{Anomalies among $\mathrm{C}-$-complex NEOs}

According to the currently accepted scenario of near-Earth objects (NEOs) origin, two main source regions have to be taken into account. The Main Belt (MB), from which about $80 \%$ of NEOs have been originated, according to the current estimates, is the principal one; the outer Solar System (SS) is by far less important $(\sim 20 \%)$. Dynamical models (Morbidelli et al. 2002) also indicate which are the injection main "channels", i.e. the regions from which various dynamical effects can cause a strong and relatively 
fast evolution of the orbital parameters, causing the injection of some -or most- bodies into the near-Earth region or into a dynamical trail with the same final destination. Seven main channels are listed: for most NEOs it is possible to estimate the probability of having been originated from each of them. The scenario presented in the above quoted paper and in the following improvements is capable to explain many observed features, and is considered as rather reliable.

The analysis of primitive NEOs (i.e. belonging to C-complex; up to now 35 objects belong to this complex) is somehow problematic. Due to a lack of distinctive spectral features, the real composition of these objects is not well understood, and there is a sort of "degeneracy" among C-complex MB-related NEOs and outer SS-related ones: in other words, on a spectral basis, C-complex NEOs may originate from the MB or the outer SS. Notice that this does not happen for the -by far more numerous- $\mathrm{S}-$ complex NEOs. Indeed, with an average probability for the JFC channel of about $4 \%$, the majority of Ccomplex NEOs are thought to originate from the MB (see Fig. 1). Notice that, according to the discussion presented by Lazzarin et al. (2006) the taxonomic separation among objects with relatively featureless spectra might be not completely meaningful, being mainly due to a different reddening history.

The C-complex NEOs are different - at least for a pair of statistical properties- from the analogue MBAs:

(a) Aqueous alteration signatures have been observed on several MBAs (about $40 \%$ of $\mathrm{C}$-complex MBAs are aqueously altered). In the visible range the hydration signatures consist of several absorption bands, the $0.7 \mu \mathrm{m}$ band being the most prominent. It must be noted that this feature is not easily detected in the case of a low $\mathrm{S} / \mathrm{N}$ value of the spectrum, such as in the case of some small MBAs or NEOs. The hydration percentage, among MBAs, does not depend on the size (see, however, the discussion in the following). We cannot find a systematic effect connected to the family membership. Some families (i.e. (24) Themis) are characterized by a very small percentage of hydrated bodies, some others (i.e., (668) Dora) behave in the opposite way, without any apparent rule. Note also that some Themis family members show an anomalous quasi-cometary activity (Hsieh \& Jewitt 2006). The combination of this feature with the overall scarcity of hydrated bodies in the same family might be strongly constraining for a theoretical explanation. The properties of the C-complex MBAs and the distribution of origin channels (Fig. 1) should entail that a considerable fraction (a conservative estimate yields $>22 \%$, i.e. something as $>8 \pm 1$ out of 35 objects) of NEOs should be hydrated. In reality only 2 NEOs show clear hydration features. Notice that the above estimate takes into account the actual abundance of hydrated bodies in the proximity of the channels.

(b) Another difficulty comes from the analysis of space weathering properties. While the C-complex MBAs redden with increasing exposure to Sun ion flux, with a behavior which is rather similar to what happens to silicate-rich MBAs (and NEOs), the Ccomplex NEOs behave in the opposite way (Fig. 2). This result stands even if we exclude in the sample all the NEOs which may have been originated from the outer SS.

\section{Discussion}

A number of tentative explanations to the former problems can be suggested, but none (until now) completely satisfactory. The full range of possibilities will be discussed in detail in a forthcoming paper.

(a) Hydrated NEOs actually do not exists: only non-hydrated bodies enter the channels and become NEOs. This would be the case if hydrated bodies would not exist among very small asteroids. We remind that all $\mathrm{C}$-complex NEOs have diameter (D) less than 
$10 \mathrm{~km}$. Only few MB objects have been observed in this size range; among them 10 nonhydrated and 2 hydrated MBAs, with $\mathrm{D}<10 \mathrm{~km}$, have been observed so far. The present values are consistent both with the independence of hydration properties on size and with a deficit of hydrated small asteroids. In principle, the dehydration might be induced by high temperature shocks which originate the small fragments, and, in particular, the NEOs. On the other hand the mineralogical product of water alteration has been found on many primitive meteorites (CCs). CCs of type 1 contain limited, but significant, evidence of aqueous alteration; while types 2 and 3 ubiquitously present products of aqueous alteration. If these products are so common on CC meteorites, why could hydration be absent among $\mathrm{C}$-complex NEOs? Both the observational evidence and the theoretical explanation are severely challenged.

(b) Another possibility is that the percentage of NEOs coming from the channels at the extremities of the alteration region (Hungaria (H in Fig. 1), $\nu_{6}$, JFC; where hydrated MBAs are not present) is larger than expected. However, $\mathrm{H}$ and $\nu_{6}$ can be excluded because of the paucity of $\mathrm{C}$-complex asteroids in their neighborhood. A larger than expected fraction of primitive NEO should come from the outer SS. However, we have no dynamical reason to cast doubts on a model which seems to work properly.

(c) Another possibility is that hydrated asteroids are indeed injected into the usual delivery channels and evolve to NEOs, but during this evolution they have lost the visible spectral signatures of aqueous alteration. What could be the cause(s) for this behavior? Indeed, Marchi et al. (2006b) have shown that planetary close encounters may affect the surface properties of S-complex NEOs. May a similar process be responsible of the lack of aqueous alteration among NEOs? Note that, in this case, the mixing of surface material with underlying layers will not cancel the alteration features, unless the body is assumed as not homogeneous, with its aqueous alteration depending on the depth. Considering that NEOs are small, and that the alteration is generally believed to be a large scale effect, it is difficult to support this suggestion. A different mechanism should be at work. Some experimental works (Hiroi et al. 1996) suggested that the heating of hydrated material, at a temperature in the range $400-600 \mathrm{~K}$, would cancel out the visible spectral signatures of aqueous alteration. A possible source of heating could be the Sun radiation. For very dark objects (such as $\mathrm{C}-$ complex bodies are), the sub-solar temperature (namely the maximum temperature achievable) scales as $5777\left(R_{\odot} / a\right)^{1 / 2} \mathrm{~K}$, which entails a temperature of about $400 \mathrm{~K}$ at $1 \mathrm{AU}$ from the Sun. Therefore, Sun heating may represent an efficient mechanism for the surface dehydration of NEOs with perihelion distance of the order or less than about 1 AU. The Sun-heating mechanism is, for the moment, the most promising one among those we discussed, and should be analysed in more detail, even with a systematic scrutiny of the dynamical properties of all the objects in the sample.

However, at the moment, the problem of explaining the strong deficit of hydrated NEOs remains open.

Moreover, the inverse behavior of the slope vs. exposure should be understood. In the case of family bodies, according to Lazzarin et al. (2006) it might be due to a sample selection effect. In this case the reason might be similar, since the sample is rather small. Or may some of the physical processes discussed above affect also the space weathering properties? In both cases we do not have at disposal any explanation, for the moment. In principle, we think that the capability to explain this anomaly together with the lack of hydrated NEOs should be the marker of a -future- inspired and successful theoretical model. 


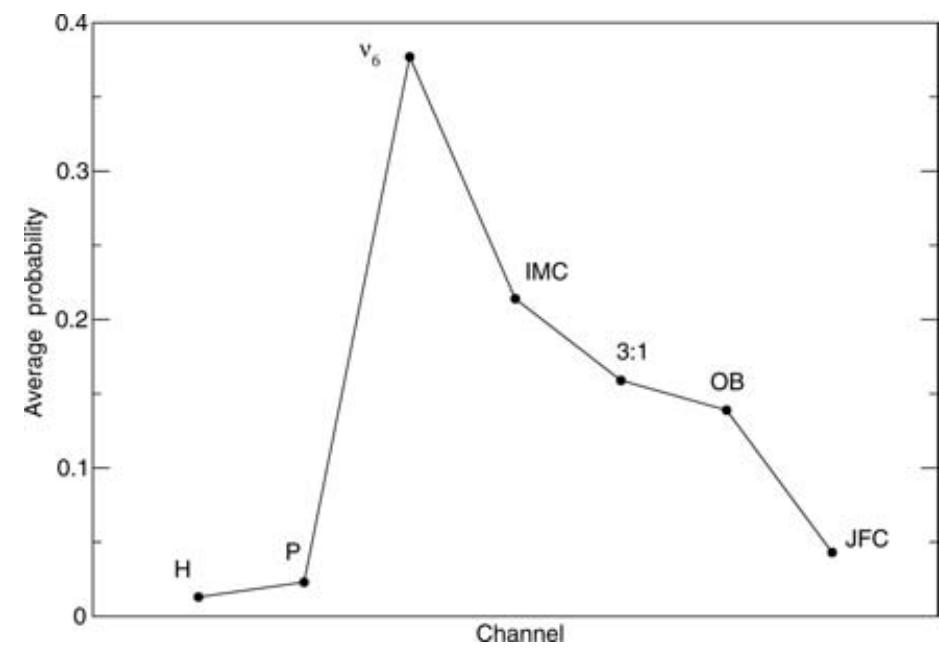

Figure 1. Average probability of origin, for all C-complex NEOs, as a function of the main different channels of origin (data by A. Morbidelli). H means Hungaria, P Phocaea, IMC, OB and JFC are respectively Mars Crossers, Outer Belt bodies and Jupiter Family Comets.

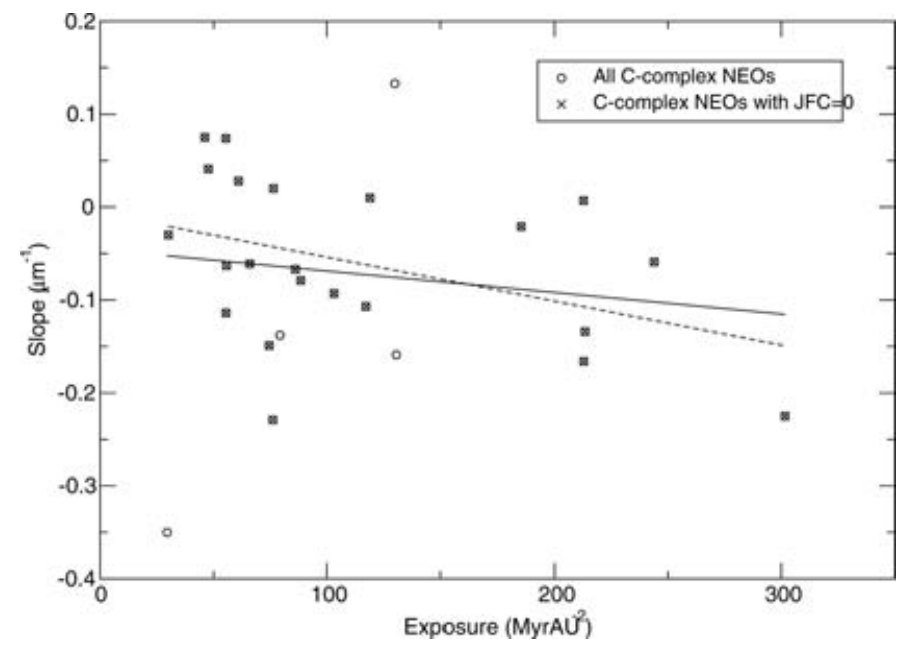

Figure 2. The slope-exposure plot for C-complex NEOs. The dashed line represents the best linear fit including only the bodies (labelled as JFC $=0$ ) which cannot, according to the channel-model (see Fig. 1), have been originated in the Jupiter-family cometary region, while the solid line corresponds to all bodies. Note that in the plot we represent only those NEOs (27 of 35) for which the slope can be estimated from the spectrum.

\section{References}

Hiroi, T., Zolensky, M.E., Pieters, C.M. \& Lipschutz, M.E. 1996, MEPS 31, 321

Hsieh, H. \& Jewitt, D. 2006, Science 312, 561

Lazzarin, M., Marchi, S., Moroz, L.V., Brunetto, R., Magrin, S., Paolicchi, P. \& Strazzulla, G. 2006, ApJ 647, L179

Marchi, S., Paolicchi, P., Lazzarin, M. \& Magrin, S. 2006a, AJ 131, 1138

Marchi, S., Magrin, S., Nesvorný, D., Paolicchi, P. \& Lazzarin, M. 2006b, MNRAS 368, L39

Morbidelli, A., Bottke W.F., Froeschlé, Ch. \& Michel, P. 2002, in: W.F. Bottke, A. Cellino, P. Paolicchi \& R.P. Binzel (eds.), Asteroids III (Tucson: University of Arizona Press), p. 619 